\title{
Formulación para reducir las palpitaciones cardiacas, una receta médica de mediados del siglo xvill en el Nuevo Reino de Granada
}

\author{
Prescription to reduce heart palpitations, a mid-18 ${ }^{\text {th }}$ century prescription \\ in the New Kingdom of Granada
}

\section{Jorge Uribe-Vergara1*, Ignacio Briceño-Balcázar', Julio C. Martínez-Lozano ${ }^{1}$, Carlos Pacheco-Cuentas ${ }^{1}$, Eduardo Tuta-Quintero', Alejandro Rueda-Rodríguez ${ }^{1}$ y Alberto Gómez-Gutiérrez²}

${ }^{1}$ Facultad de Medicina, Universidad de la Sabana, Chía; ${ }^{2}$ Instituto de Genética Humana, Facultad de Medicina, Pontificia Universidad Javeriana. Bogotá, Colombia

\section{Resumen}

A lo largo de la historia, el ser humano ha utilizado plantas para prevenir, aliviar y curar enfermedades. Cabe destacar que durante mucho tiempo se desconoció el mecanismo por el cual su uso era beneficioso, lo que hacía de la fitoterapia una ciencia netamente empírica. Las recetas médicas eran consideradas un avance médico significativo, resultado de conocimientos y prácticas traídas desde el viejo continente. Pese a la carencia de estudios locales, la literatura médica de la época permitió utilizar la biodiversidad de América para el desarrollo, investigación y uso de nuevos esquemas fitoterapéuticos con dosificaciones establecidas e indicaciones de uso, incluso un lugar específico de dispensación, ampliando aún más las posibilidades terapéuticas. La receta médica que se expone en este artículo era empleada en el manejo de las palpitaciones cardiacas, síntoma frecuente aún en la actualidad, descrito como golpes en tórax y/o cuello, subyacente en diversas enfermedades cardiacas y no cardiacas. La receta para las palpitaciones del archivo histórico Cipriano Rodríguez Santa María es una mezcla de agentes herbarios que, al realizar una revisión en la literatura médica, evidencian efectos antiinflamatorios, ansiolíticos y antioxidantes entre otros, sustentando un posible efecto beneficioso en las palpitaciones cardiacas. Debido a la ausencia de información sobre la posología, seguridad en su uso, contraindicaciones y posibles efectos adversos, es subestimado su potencial uso en aquel entonces para el control de las palpitaciones o como unos agentes fitoquímicos dirigidos para tratar enfermedades causantes de dicho síntoma.

Palabras clave: Palpitaciones. Historia del siglo XIX. Síntomas. Medicina herbal.

\begin{abstract}
The human being, throughout history, has used plants to prevent and cure diseases. It is important to know that for a long time, the mechanism through which those plants worked was unknown, making herbal medicine a purely empirical science. Medical prescriptions in the 19th century in the Kingdom of Nueva Granada were considered a significant medical advance,
\end{abstract}

Correspondencia:

*Jorge Uribe-Vergara

E-mail: jorgeuribe7@ hotmail.com

DOI: 10.24875/ACM.19000268

1405-9940/๑ 2019 Instituto Nacional de Cardiología Ignacio Chávez. Publicado por Permanyer. Este es un artículo open access bajo la licencia CC BY-NC-ND (http://creativecommons.org/licenses/by-nc-nd/4.0/).
Disponible en internet: 29-01-2020 Arch Cardiol Mex. 2020;90(2):138-143 www.archivoscardiologia.com 
as a result of knowledge and medical practices in the old continent. Medical literature of the time achieved, despite the lack of studies, the development of new schemes with exact dosages and new therapeutic possibilities. The medical prescription presented in this article was used in the management of heart palpitations, a frequent symptom nowadays, described as thoracic and/or neck beating, underlying various cardiac and non-cardiac diseases. The recipe for the palpitations of the historical archive "Cipriano Rodríguez Santa María" is a mixture of herbal agents that, as reviewed in the medical literature, showed to have anti-inflammatory, anxiolytic, and antioxidant effects, among others, allowing a beneficial effect on cardiac palpitations. Due to the lack of information on the posology, safety in its use, contraindications and possible adverse effects, its potential use should have been underestimated at that time for the control of palpitations or as phytochemical agents directed to treat diseases causing this symptom.

Key words: Palpitations. History of $19^{\text {th }}$ century. Symptoms. Herbal medicine.

\section{Introducción}

El ser humano desde sus inicios aprendió a aprovechar las particularidades de animales, minerales y vegetales con fines curativos; este conocimiento se desarrolló de manera empírica a base de ensayo y error y seguramente como producto de una gran capacidad de observación. De esta manera se fue construyendo el conocimiento basado en el efecto, pero sin la base científica que explicara las diferentes propiedades de los elementos utilizados. Este documento pretende abordar una receta del siglo XVIII para realizar una aproximación a la base científica que permita explicar su mayor o menor éxito en los tratamientos para los que se proponía.

El empleo ancestral de plantas medicinales se atribuye a la experiencia de indígenas, protomédicos y médicos de distintas épocas de la medicina neogranadina, que fueron capaces de percibir directamente en la naturaleza elementos útiles a la terapia que confronta la enfermedad, permitiendo el desarrollo de recetas médicas utilizadas ampliamente en el Nuevo Reino de Granada para el manejo de enfermedades. La llegada de diferentes manuscritos traídos desde el viejo continente, unidos a los conocimientos de nativos en América basados en su propia flora, enriquecieron la práctica médica. En este proceso se dio la formación de médicos locales liderada por José Celestino Mutis (1732-1808), pionero en la creación de cátedras de medicina en Santafé. Mutis logró unir la medicina y la botánica dando importancia a la fitoterapia que, en aquel entonces, era la fuente principal de salud con agentes herbarios para el tratamiento de enfermedades leves o moderadas, así como coadyuvante en las enfermedades crónicas como el insomnio o la depresión, entre otros ${ }^{1}$.

Las palpitaciones son un síntoma frecuente en la consulta médica, refiriéndose a una sensación anormal e incómoda de los latidos o contracciones enérgicas del corazón, descritas en tórax y/o cuello como golpes o tirones que en ocasiones son asociadas por lo pacientes a una sensación «de muerte». Estas son un síntoma subyacente en diversas enfermedades cardiacas de naturaleza eléctrica o estructural y no cardiacas $^{2-4}$. A mediados del siglo XVIII los avances médicos neogranadinos estaban influenciados por conocimientos y prácticas esencialmente españolas, pese a ello se desconocía la fisiopatología de enfermedades asociadas a las palpitaciones.

El archivo histórico Cipriano Rodríguez Santa María de la Universidad de La Sabana, posee diferentes recetas médicas del siglo XVIII y comienzos del XIX. Entre estas, conserva una receta sobre prácticas hace más de dos siglos, cuyo fin era el tratamiento de las palpitaciones (Fig. 1). Una mirada histórica al pasado nos permite comprender las bases científicas que sustentan la fitoterapia, en aquel entonces una práctica médica totalmente empírica.

\section{Material y métodos}

Revisión histórica, cultural y científica, basada en el documento denominado «Receta para las palpitaciones» conservado en el archivo histórico Cipriano Rodríguez Santa María de la Universidad de La Sabana. Se realizó una búsqueda en las bases de datos ScienceDirect, ClinicalKey, Ovid, PubMed y SciELO, utilizando los términos «palpitaciones», «fitoterapia», «history», «fitoquímicos», «history of $19^{\text {th }}$ century»; sin límite de tiempo e idioma inglés y español. Una vez recolectada la información, se realizó una revisión de la definición moderna de palpitaciones, fisiopatología y tratamiento, así como de los avances médicos de los siglos XVIII y XIX en el Nuevo Reino de Granada. Simultáneamente se describieron los fitoquímicos encontrados en la receta y su posible mecanismo de acción en el manejo de las palpitaciones o enfermedades subyacentes. 


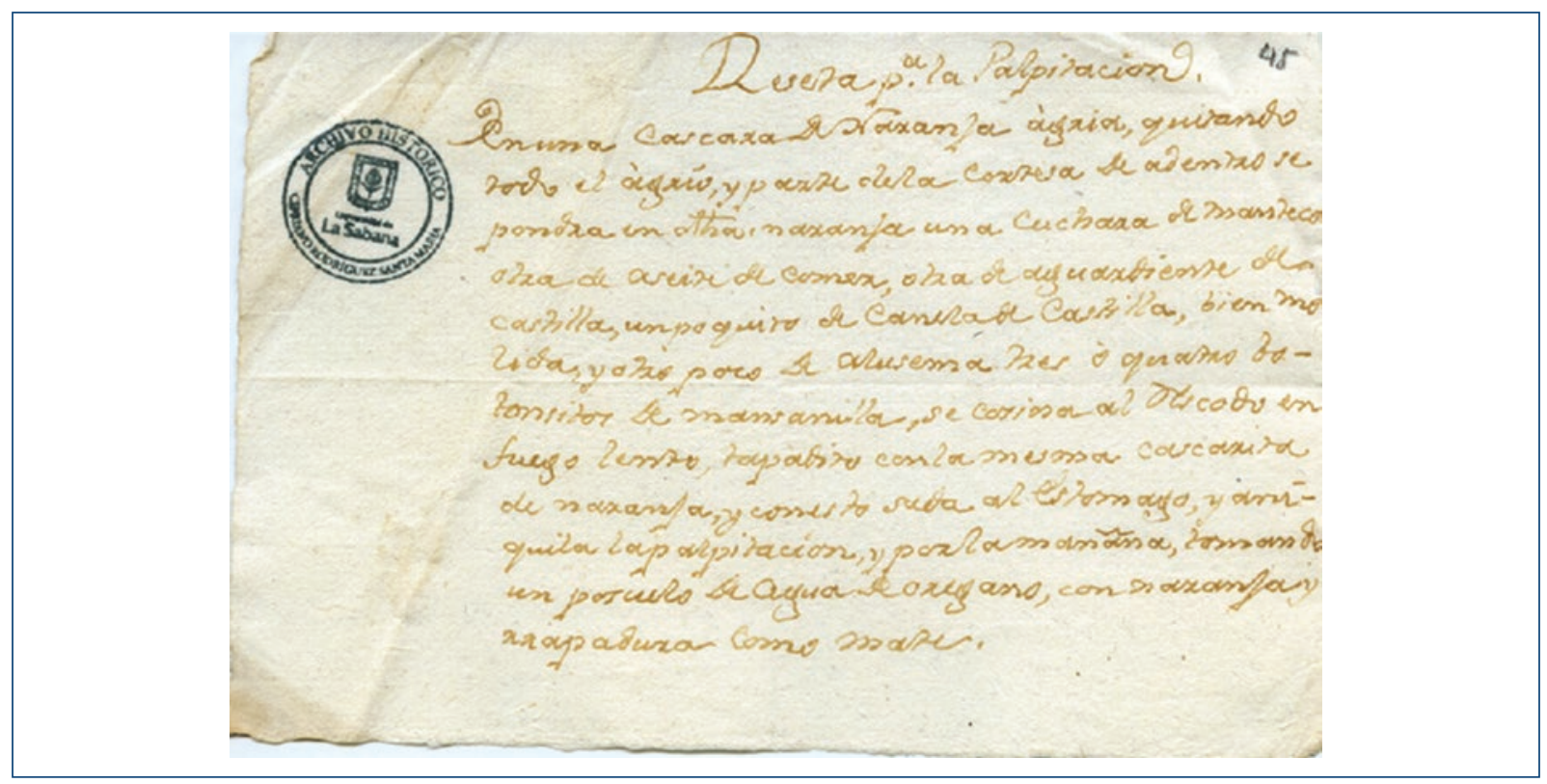

Figura 1. Receta para la palpitación (fuente: Archivo Histórico Cipriano Rodríguez Santamaría. Biblioteca Octavio Arizmendi Posada, Universidad de La Sabana. Caja 10, carpeta 2, folio 45 recto).

\section{Transcripción del documento fuente}

«Reseta para la palpitacion (se conserva la ortografía original del manuscrito).

En una cascara de Naranja àgria, quitando todo el àgrio, y parte dela cortesa de adentro se pondra en dicha naranja una cuchara de manteca otra de aseite de comer, otra de aguardiente de castilla, un poquito de Canela de Castilla, bien molida y otro poco de azucema tres ò quatro botonsitos de mansanilla, se cosina al resco[I]do en fuego lento, tapadito con la mesma cascarita de naranja, y con esto suda al estomago, y aniquila la palpitacion, y por la mañana, tomando un poquito de agua de oregano, con naranja y raspadura como mate».

\section{Resultados}

Esta receta de mediados del s. xVIII se prescribía para reducir las palpitaciones cardiacas, en esa época empezaban a circular textos médicos de terapéutica que contribuían al desarrollo de la medicina en el Nuevo Reino de Granada. En la receta se expone minuciosamente un tratamiento con una variedad de componentes herbales, pero no se define o describe el término palpitación, lo que hubiera podido generar confusión al momento de su empleo. Los avances médicos de la época basados en la fitoterapia tampoco permitían un respaldo científico basado en la evidencia, por lo que se reconocía de una manera exclusivamente empírica y deductiva la actividad biológica de los componentes fitoquímicos, que sin embargo tenían efectos beneficiosos para la salud, siempre que este tratamiento se convirtiera en un hábito por un largo periodo de tiempo, sin ser esta una cura de enfermedades sino un promotor de estilo de vida saludable.

De acuerdo con los conocimientos modernos al iniciar el siglo xxI, las palpitaciones ocupan un puesto importante entre los síntomas más comunes como motivo de consulta a médicos generales, cardiólogos o servicios de urgencias, solo precedidas por el dolor torácico ${ }^{3,4}$, obedeciendo a una sensación descrita en tórax y/o cuello como golpes, movimientos o tirones, en ocasiones asociadas por lo pacientes a una sensación «de muerte». Las palpitaciones tienen una amplia gama de patologías subyacentes, cardiacas y no cardiacas, siendo más frecuentes en las enfermedades eléctricas del corazón, generalmente con una duración de unos segundos a un par de minutos producidas por extrasístoles (auriculares/ventriculares), o un cuadro clínico de minutos, horas o días asociado a taquiarritmias sostenidas (supraventriculares/ventriculares). Por esta razón, el paciente suele presentarse asintomático a la consulta haciendo obligatorio para el diagnóstico de la etiología una evaluación semiológica completa, preguntando 
sobre el síntoma su forma de inicio, frecuencia, duración, irradiación de la sensación, desencadenantes y asociados, sin olvidar indagar por antecedentes patológicos, tóxicos y familiares ${ }^{5}$. Los estudios paraclínicos empleados son seleccionados gracias a la historia clínica del paciente y experticia clínica del médico tratante; el electrocardiograma debe ir incluido en la escalera diagnóstica debido a su fácil accesibilidad y su alto desempeño como método diagnóstico. En pacientes seleccionados se puede considerar un monitor Holter de 24 horas $^{5,6}$.

Los mecanismos fisiopatológicos de las palpitaciones no son claros, se ha descubierto su relación con receptores sensoriales en miocardio y pericardio, mecanorreceptores y barorreceptores periféricos junto a una activación del sistema simpático por vías aferentes. Debido a que las palpitaciones pueden ser causadas por múltiples condiciones como arritmias o alteraciones estructurales cardiacas, desórdenes psicosomáticos, enfermedades sistémicas como anemia, consumo de medicamentos o sustancias psicoactivas; la fisiopatología de dichas enfermedades varía entre ellas, haciendo aún más difícil la explicación fisiopatológica exacta de este síntoma. Cabe aclarar que las palpitaciones fisiológicas son percibidas y asociadas por el paciente en contextos como la actividad física intensa o emociones fuertes, vista como una respuesta del cuerpo a un desafío generalmente de corta duración ${ }^{5,6}$.

Las palpitaciones se asocian a una baja tasa de mortalidad; sin embargo, el pronóstico recae sobre la enfermedad subyacente y comorbilidades asociadas del paciente. El manejo de las palpitaciones debe dirigirse a la condición médica de base ${ }^{6}$.

Pese a que los componentes de esta receta colonial son resultado de conocimientos y prácticas altamente utilizadas por médicos en el siglo xVIII, estos carecen de evidencia científica actual que respalde sus efectos terapéuticos sobre el síntoma o enfermedad asociada, evidencia sobre seguridad, contraindicaciones y posibles efectos adversos en población especial como longevos, niños y mujeres gestantes. Con el paso del tiempo, y gracias a los avances científicos, se encontró una relación entre una alimentación saludable, agentes fitoquímicos como los incluidos en la receta descrita, disminuyendo así el impacto fisiológico de diversas enfermedades ${ }^{7,8}$.

\section{Propiedades fisicoquímicas}

Se presenta una revisión de las propiedades fitoquímicas de cada ingrediente utilizado en la receta colonial, explicando un posible mecanismo de acción y cómo este podría ser útil para el manejo de las palpitaciones de forma directa o indirecta.

\section{Cáscara de naranja (Citrus sinensis)}

Efecto antioxidante en los flavonoides como los glucósidos de flavona, las flavonas y los flavonoles, encontrados por cromatografía de capa fina

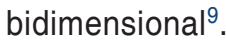

\section{Manteca (margarina)}

Es una importante fuente de ácidos grasos trans, mientras que la mantequilla es alta en grasas saturadas. En consecuencia, se utiliza como un agente eventualmente saborizante con los demás ingredientes de la receta, sin propiedades fitoquímicas que expliquen un beneficio para las palpitaciones.

\section{Aceite de cocina de oliva (Olea europaea)}

Se ha evidenciado un efecto protector en contra de la inflamación en modelo animal debido a las distintas clases de fenoles hidrofílicos, hidroxitirosol y derivados del aglicón de oleuropeína, dimetil-oleuropeína y ligstrósido presentes en la fruta de olivo. Entre estos efectos protectores destaca la reducción de la peroxidación de fosfolípidos en liposomas, protección de la oxidación de las lipoproteínas de baja densidad, reducción del daño oxidativo de los eritrocitos humanos y cambio de bases nitrogenadas por los peroxinitritos, junto a una reducción de la producción de radicales libres en la matriz mitocondrial ${ }^{10}$.

\section{Canela de Castilla (Cinnamomum zeylanicum)}

La evidencia in vitro e in vivo en modelos animales explica propiedades fitoquímicas vasodilatadoras al inhibir el calcio extracelular a través de canales sensibles al voltaje tipo $\mathrm{L}$, permitiendo posicionarlo como un antihipertensivo natural, además este ingrediente parece reducir la glucemia y el colesterol sérico ${ }^{11}$.

\section{Aguardiente de Castilla}

El alcohol en dosis de bajas tiene efectos beneficiosos en la presión arterial y reactantes de fase aguda como la proteína $C$ reactiva, simultáneamente aumenta la tolerancia del miocardio a una lesión isquémica, 
mecanismo conocido como «precondicionamiento isquémico» ${ }^{12}$.

\section{Manzanilla (Matricaria recutita)}

La apigenina (5,7'4-trihidroxiflavona), molécula aislada en esta planta con alta afinidad por lo receptores del ácido gamma-aminobutírico, que se une de manera competitiva sin actividad sobre receptores muscaríni$\cos y$ adrenérgicos (alfa 1), ejerciendo un efecto ansiolítico al reducir las corrientes de cloro de forma dependiente de la dosis, similar a las benzodiacepinas. Se demostró de igual manera en estudios en modelos animales un beneficio sobre la tensión arterial y el perfil lipídico ${ }^{13}$.

\section{Azucena (Lilium lancifolium)}

Planta tradicional china con efecto sobre macrófagos, aumentando su actividad fagocítica y fosforilación de citocinas proinflamatorias disminuyendo la quimiotaxis y con ello la respuesta inmunológica ${ }^{14}$.

Luego de esta breve descripción sobre los mecanismos de acción de dichos agentes herbarios incluidos en la receta en estudio se puede postular su posible efecto beneficioso sobre las palpitaciones o enfermedades subyacentes. Sin embargo, la ausencia de evidencia suficiente en el ser humano hace necesaria una mayor investigación en este modelo para determinar su efecto específico e impacto terapéutico.

\section{Discusión}

En los siglos XVII y XIX, el hablar de medicina era sinónimo de conocimientos empíricos y científicos traídos de Europa, permitiendo una recopilación de recetas médicas por parte de españoles y nativos americanos, que con el pasar del tiempo aumentaría su uso en el Nuevo Continente. Una de ellas, empleada para el manejo de las palpitaciones, estaba compuesta por fitoquímicos con propiedades antioxidantes, antiinflamatorias y ansiolíticas, pudiendo lograr hasta cierto punto el control de las palpitaciones o algún beneficio en enfermedades subyacentes como un antihipertensivo natural. Sin embargo, una vaga información en parámetros o condiciones de su uso y descripción del síntoma, desacredita su potencial empleo en aquella época para el control de las palpitaciones o como un agente fitoquímico dirigido para enfermedades causantes de dicho síntoma. Se destaca la labor hecha por los médicos o boticarios, quienes eran los encargados de prescribir una medicina herbal basados en las recetas, seguir la evolución del paciente y lograr una efectividad en el alivio de la sintomatología. Esto sucedía en un tiempo de la historia en la cual la iglesia y la corona decidían quienes debían ejercer la medicina, pese que esto era un acto de valor debido a la ausencia de salubridad pública y el alto riesgo biológico al que se veía enfrentado el médico o boticario al tener una relación estrecha con el paciente.

\section{Conclusión}

Hasta el siglo xVII, la humanidad había alcanzado un desarrollo moderado en la comprensión de diversos aspectos asociados a la mayoría de los fenómenos médicos. A partir de la segunda mitad del siglo xVII y hasta comienzos del siglo XIX, se desarrollaron varias teorías médicas que disputarán un lugar a las ideas de Galeno que habían estado vigentes por más de 1,500 años. Estas teorías dieron lugar a diferentes conceptos de enfermedad y por consiguiente a la modificación de la terapéutica utilizada hasta entonces. Todo este movimiento tuvo lugar bajo la influencia que en ese momento determinaron diferentes corrientes de pensamiento filosófico en el desarrollo de la ciencia médica de la época. Esta influencia se refleja en el origen de las primeras prácticas médicas basadas inicialmente en dos maneras diferentes de abordar el problema: el primero es el empirismo primitivo, caracterizado por el pragmatismo y el uso de medicamentos y tratamientos obtenidos de la naturaleza, y el segundo la visión médica influenciada por la corriente mágico-religiosa, que intentaba explicar la enfermedad desde el misticismo y las creencias institucionalizadas por la iglesia.

Aunque en el Virreinato de la Nueva Granada el conocimiento de la medicina se desarrolló de manera muy similar al europeo, este estuvo marcado primordialmente por el encuentro de tres mundos, el indígena, el español y el africano, los cuales, partiendo de una concepción de la polaridad salud-enfermedad, eran similares en función de una gran carga cultural, social, moral y emotiva relacionada con el castigo, el pecado, la degeneración y los vicios, como causa o razón de la patología.

El presente trabajo busca aportar al proceso de construcción de la historia de la medicina nacional, partiendo del análisis de documentos que recrean desde diferentes ángulos y patologías el abordaje médico del momento, tratando de encontrar una explicación integral y documentada de la práctica y el conocimiento médico local predominante en ese momento de la historia. 


\section{Agradecimientos}

Los autores expresan agradecimiento a Marcela Revollo Rueda, directora del Archivo Histórico de la Biblioteca Octavio Arizmendi Posada de la Universidad de La Sabana.

\section{Financiamiento}

El proceso de investigación y publicación fue financiado por las universidades a las cuales están afiliados los autores.

\section{Conflicto de intereses}

Los autores declaran no tener ningún conflicto de intereses.

\section{Responsabilidades éticas}

Protección de personas y animales. Los autores declaran que para esta investigación no se han realizado experimentos en seres humanos ni en animales.

Confidencialidad de los datos. Los autores declaran que en este artículo no aparecen datos de pacientes.

Derecho a la privacidad y consentimiento informado. Los autores declaran que en este artículo no aparecen datos de pacientes.

\section{Bibliografía}

1. Baulies G, Torres R. Actualización en fitoterapia y plantas medicinales. FMC, Form méd contin aten prim. 2012;19(3):149-60.

2. Pascual $\mathrm{M}$, Valverde $\mathrm{M}$, Zamorano JL. Protocolo de tratamiento del paciente con palpitaciones. Rev Educ Super. 2017;12(39): 2333-4.

3. Mateo-Martínez A, Villegas-García M, Sánchez-Martínez M. Abordaje de las palpitaciones y la taquicardia. FMC, Form méd contin aten prim. 2015;22(3):119-31.

4. Frank R, Hidden-Lucet F. Palpitaciones. EMC - Tratado Med. 2015; 19(15):1-6.

5. Álvarez-Gaviria M, Herazo-Bustos C, Mora G. Enfoque semiológico de las palpitaciones. Rev Fac Med. 2014;62(1):119-30.

6. Raviele A, Giada F, Bergfeldt L, Blanc JJ, Blomstrom-lundqvist C, Mont L, et al. Management of patients with palpitations: a position paper from the European Heart Rhythm Association. Europace. 2011;13: 920-34.

7. Fuentes-Jiménez F, López-Miranda J. Utilización de productos naturales de la dieta con efectos bioactivos con fines terapéuticos a nivel poblacional. Clínica e Investig en Arterioscler. 2012;24(1):1-2.

8. Martínez-Navarrete N, Mar M, Martínez J. Actividad dietética. Los compuestos bioactivos de las frutas y sus efectos en la salud. Act Diet. 2008;12(2):64-8.

9. Anagnostopoulou MA, Kefalas P, Papageorgiou VP, Assimopoulou AN, Boskou D. Radical scavenging activity of various extracts and fractions of sweet orange peel (Citrus sinensis). Food Chem. 2006; 94(1):19-25.

10. Servili M, Selvaggini R, Esposto S, Taticchi A, Montedoro G, Morozzi G. Health and sensory properties of virgin olive oil hydrophilic phenols: agronomic and technological aspects of production that affect their occurrence in the oil. J Chromatogr A. 2004;1054:113-27.

11. Ranasinghe $P$, Pigera $S$, Premakumara GAS, Galappaththy P. Medicinal properties of "true" cinnamon (Cinnamomum zeylanicum): a systematic review. BMC Complement Altern Med. 2013;13(1):275.

12. Gardner JD, Mouton AJ. Alcohol effects on cardiac function. Compr Physiol. 2015;5:791-802.

13. Awaad AA, El-Meligy RM, Zain GM, Safhi AA, Al Qurain NA, Almoqren SS, et al. Experimental and clinical antihypertensive activity of Matricaria chamomilla extracts and their angiotensinøconverting enzyme inhibitory activity. Phytother Res. 2018;32(8):1564-73.

14. Pan G, Xie Z, Huang S, Tai $Y$, Cai $Q$, Jiang W, et al. Immune-enhancing effects of polysaccharides extracted from Lilium lancifolium Thunb. Int Immunopharmacol. 2017;52:119-26. 